Case Report

\title{
General anesthesia for a patient with deletion 6q syndrome in addition to laryngomalacia undergoing dental treatment
}

\author{
Keiko Fujii-Abe, Mami Sasao and Haruhisa Fukayama \\ Department of Dental Anesthesiology, School of Dental Medicine, Tsurumi University, Yokohama, Japan
}

(Received 3 June and accepted 2 October 2008)

\begin{abstract}
Chromosome 6 deletions are very rare $(1,2)$, and deletion $6 q$ syndrome is clinically characterized by mental and/or neuromotor retardation and microcephaly (3). Other alterations frequently observed are decreased biparietal diameter, hypertelorism, hypotelorism, absent eyebrows, prominent eyes with ptosis, receding chin, dysmorphic ears, large extremities, prominent nasal bridge, long philtrum, epicthus, strabismus, and micrognathia (35). Laryngomalacia is the most common congenital laryngeal anomaly and the most frequent cause of stridor in infants (6-11). We report the case of a 14-yearold male patient with both deletion $6 q$ syndrome and laryngomalacia, who we treated for multiple dental caries. He had a medical history of tracheotomy at age 11 years for laryngomalacia, and has suffered from epileptic attacks and aspiration pneumonia over the last 2 and 6 years, respectively. Since he was mentally retarded and in a poor respiratory state, dental treatment under general anesthesia was scheduled in our hospital. General anesthesia was induced and maintained using $30 \%$ nitrous oxide and 1-3\% sevoflurane in oxygen through the tracheotomy tube. Pre- and intraoperative endotracheal suction improved the condition of both lungs markedly and the procedures were uneventful and completed in $2 \mathrm{~h}$ and $58 \mathrm{~min}$. (J. Oral Sci. 50, 493-495, 2008)
\end{abstract}

Correspondence to Dr. Keiko Fujii-Abe, Department of Dental Anesthesiology, School of Dental Medicine, Tsurumi University, 2-1-3, Tsurumi, Tsurumi-ku, Yokohama, Kanagawa 230-8501, Japan

Tel: +81-45-580-8342

Fax: +81-45-573-9599

E-mail: fujii-keiko@tsurumi-u.ac.jp
Keywords: laryngomalacia; deletion 6q syndrome; dental treatment; general anesthesia.

\section{Case Report}

The patient was a 14-year-old boy, weighing $30 \mathrm{~kg}$ and $128 \mathrm{~cm}$ in height. He was born after a 36-week pregnancy by emergency caesarian section owing to a faint heart sound, weighing $1,720 \mathrm{~g}$. At birth, he showed polydysplasia, such as duodenal atresia, patent ductus arteriosus, lateroflexion of the second and fourth toes and ocular hypertelorism. The patient was diagnosed as having deletion 6q syndrome. The points of breakage of chromosome were not identified in this case and his family medical history was unremarkable.

He had two operations for duodenal atresia at age 3 days and for patent ductus arteriosus at age 3 months. He also underwent tracheotomy at age 11 years for laryngomalacia. His medical history includes epilepsy in the last two years and frequent aspiration pneumonia starting from age 8 years as a result of his eating difficulties.

For the dental treatment of multiple caries, systemic management including general anesthesia was scheduled at our hospital. We applied general anesthesia for his dental treatment because he had mental retardation and required treatment for multiple caries under stable respiratory conditions. His attending pediatrician agreed with the decision to perform dental treatment under general anesthesia.

Preoperative chest X-ray revealed marked radiopaque areas in the superior, middle, and inferior lobes of both lungs, and oxygen saturation in room air was $90-92 \%$. Preoperative chest X-ray showed signs indicating an air bronchogram, which indicated pneumonitis. Peripheral pulmonary alveolar sounds were moist and included rales. 
Blood test showed slight anemia with hemoglobin at 11.3 $\mathrm{g} / \mathrm{dl}$. His current drug regimen included theophylline, ambroxol hydrochloride, tulobuterol, L-carbocisteine, sulfamethoxazole trimethoprim, sodium valproate and tulobuterol tape.

There were several problems related to the management of this patient; mental retardation, low oxygen saturation (90-92\%), radiopaque lungs, moist breath sounds with rales, epilepsy, tracheostomy, slight anemia and dysphagia.

He was fasted for $9 \mathrm{~h}$. Premedication was intrarectal administration of $3 \mathrm{mg}$ of bromazepam $40 \mathrm{~min}$ before the induction of general anesthesia. General anesthesia was induced using 30\% nitrous oxide and 3\% sevoflurane in oxygen through the tracheostomy tube. After adequate depth of anesthesia was obtained, a mass of yellow glutinous secretion was removed through the tracheotomy tube. An antibiotic agent, cefazolin sodium, was administered perioperatively to prevent respiratory crisis. General anesthesia was maintained using 30\% nitrous oxide and 1-3\% sevoflurane and oxygen in combination with local anesthetic, $2 \%$ lidocaine. Suction was repeated $45 \mathrm{~min}$ after the start of surgery. His respiratory condition was markedly improved by endotracheal suctions, which was recognized by postoperative chest X-rays.

His four residual deciduous teeth were extracted, three decayed teeth were filled using composite resin (lower left first molar and upper bilateral first anterior teeth), and preventative filling was carried out for other permanent molar teeth. Furthermore, dental calculi were depleted. Dental treatment was successfully completed in $2 \mathrm{~h}$ and 58 min without any intraoperative complications. He passed urine and ingested food $30 \mathrm{~min}$ and $5 \mathrm{~h}$ after surgery, respectively. There were no complications postoperatively, and the patient was discharged from the hospital the next day.

\section{Discussion}

Chromosomal imbalances involving chromosome 6 in humans are uncommon (4) and chromosome 6 deletions are very rare $(1,2)$. The most striking features are severe mental retardation and facial dysmorphisms $(4,12)$. Oliveira-Duarte et al. reported that deletion $6 \mathrm{q}$ syndrome is clinically characterized by mental and/or neuromotor retardation and microcephaly (3). Other alterations frequently observed are decreased biparietal diameter, hypertelorism, hypotelorism, absent eyebrows, prominent eyes with ptosis, receding chin, dysmorphic ears, large extremities, prominent nasal bridge, long philtrum, epicthus, strabismus, and micrognathia (3-5). The features of this syndrome vary substantially $(1,2,4,5,13,14)$.

Our patient showed multiple congenital anomalies related to deletion $6 \mathrm{q}$ syndrome: mental retardation, duodenal atresia, patent ductus arteriosus, lateroflexion of toes, and ocular hypertelorism. Fortunately, these were not related to the risks of general anesthesia.

Laryngomalacia is the most common congenital laryngeal anomaly and the most frequent cause of stridor in infants (6-11), and is usually a benign condition that disappears as the child grows up, usually without requiring treatment (11). Laryngomalacia is a condition in which the laryngeal tone is weak, which results in dynamic prolapse of supraglottic tissue into the airway, causing inspiratory stridor and airway obstruction (10). Its distinctive features are collapse of the arytenoids, epiglottis and aryepiglottic folds, usually during the inspiratory phase (11).

On the other hand, causes of a difficult airway in children include congenital abnormalities that are present with varying degrees of chronic obstruction, such as laryngomalacia, glottic webs, hemangiomas, vascular rings or hypoplastic mandible (15). Smith et al. reported that infants with laryngomalacia, even mild cases, can be difficult to be anaesthetize because of difficulties in artificial manual ventilation (16).

In this case, preoperative chest X-ray revealed marked impermeability in both lungs and oxygen saturation on room air was $90-92 \%$ due to frequent aspiration pneumonia. Therefore, it is necessary to prevent respiratory complications under general anesthesia. However, his usual respiration through the tracheostomy tube and perioperative respiratory management was relatively smooth. If he had not undergone tracheostomy previously, his airway management would have been very difficult during both induction of and emergence from general anesthesia.

Our case report confirms the importance of evaluating the physical status of a patient with deletion 6q syndrome and/or laryngomalacia.

\section{References}

1. Glover G, López I, Gabarron J, Carmona JA (1988) Partial monosomy $6 \mathrm{q}(\mathrm{q} 15 \mathrm{q} 21)$ by de novo interstitial deletion. Clinical Genet 33, 308-310

2. Cote GB, Papadakuo-Lagoyanni S, Metaxotou C (1981) A de novo interstitial deletion of band q21 on chromosome 6. Ann Genet 24, 170-171

3. Oliver-D MH, Martelli-D LR, Sarquis-C T, Machado ML, Lison MP (1990) Distal monosomy of the long arm of chromosome 6 (6q25 $\rightarrow$ 6qter) inherited by maternal translocation $\mathrm{t}(6 \mathrm{q} ; 17 \mathrm{q})$. Ann Genet 33, 56-59

4. Dallapiccola B, Bricarelli FD, Quartino AR, Mazzilli MC, Chisci R, Gandini E (1978) Deletion of syndromes due to partial 6q imbalances. Acta Genet 
Med Gemellol 27, 57-66

5. Nakagome Y, Tanaka T, Hashimoto T, Kuyama M, Maruyama M (1980) Interstitial deletion 6q in a malformed boy. Ann Genet 23, 49-51

6. Cheng KS, Ng JM, Li HY, Hartigan PM (2002) Vallecular cyst and laryngomalacia in infants: report of six cases and airway management. Anesth Analg 95, 1248-1250

7. Benjamin B, Lines V (1972) Endoscopy and anaesthesia in non-infective airway obstruction in children. Anaesthesia 27, 283-291

8. Olney DR, Greinwald JH, Smith RJH, Bauman NM (1999) Laryngomalacia and its treatment. Laryngoscope 109, 1770-1775

9. Reddy DK, Matt BH (2002) Unilateral vs. bilateral supraglottoplasty for severe laryngomalacia in children. Arch Otolaryngol Head Neck Surg 127, 694-699

10. Thompson DM (2007) Abnormal sensorimotor integrative function of the larynx in congenital laryngomalacia: a new theory of etiology. Laryngoscope 117, 1-33

11. Midulla F, Guidi R, Trancredi G, Quattrucci S, Ratjen F, Bottero S, Vestiti K, Francalanci P, Cutrera
R (2004) Microaspiration in infants with laryngomacia. Laryngoscope 114, 1592-1596

12. Elia M, Striano P, Fichera M, Gaggero R, Castiglia L, Galesi O, Malacarne M, Pierluigi M, Amato C, Musumeci SA, Romano C, Majore S, Grammatico P, Zara F, Striano S, Faravelli F (2006) 6q terminal deletion syndrome associated with a distinctive EEG and clinical pattern: a report of five cases. Epilepsia 47, 830-838

13. Rivas F, Ruiz C, Rivera H, Moller M, SerranoLucas JI, Cantu JM (1986) De novo del(6)(q25) associated with macular degeneration. Ann Genet $29,42-44$

14. Eash D, Waggoner D, Chung J, Stevenson D, Martin CL (2005) Calibration of 6q subtelomere deletions to define genotype/phenotype correlations. Clinical Genet 67, 396-403

15. Hall SC (2001) The difficult pediatric airwayrecognition, evaluation, and management. Can J Anesth 48, 1-5

16. Smith TGC, Whittet H, Heyworth T (1992). Laryngomalacoa-a specific indication for the laryngeal mask? Anaeathesia 47, 910 